\title{
BAROCKE THEMATIK IN DER LYRIK DES ANDREAS GRYPHIUS.
}

i. Einige Worte über Wege, Umwege und Irrwege in der Barockforschung.

Jeder der heute, zehn Jahre nach Beendigung des zweiten Weltkrieges, einmal wieder zur Lyrik des Andreas Gryphius greift, wird mit Erstaunen feststellen, wie zeitnahe uns doch der alte schlesische Meister ist. Wir verstehen seine Worte:

Ist jemals, weil der bau der groszen welt gestanden, So grimme tyranney und greuel auch erhört?

Ist was, das nicht durch krieg, schwerdt, flamm' und spiesz zustört? (S. 66) ${ }^{1}$.

Darum ist auch das besondere Interesse an der Dichtung des Barock seit der zwanziger Jahre unseres Jahrhunderts nicht mehr zurückgegangen. Eine fieberhafte Forschertätigkeit hat sich im Laufe der Jahre entwickelt, sowohl in Europa als neuerdings auch in den Vereinigten Staaten; Untersuchungen wurden durchgeführt, die eine umfangreiche Bibliographie zum Resultat hatten, andererseits aber leider auch oft die Erkenntnis brachten, dasz man einen Um- oder Irrweg gegangen war. So scheint es uns heute möglich--bei aller Hochachtung, die wir Wölfflin als „Entdecker” barocker Stilbegriffe schuldig sind--auch ohne stilistische Kategorien an eine Untersuchung des Barockproblems herangehen und zu einem ähnlichen, wenn auch weniger schulmäszigen und abstrakten, Ergebnis kommen zu können, wie die Literaturwissenschaftler, die eine direkte bertragung Üder Wölfflinschen Kategorien unternahmen. Um eine solche Untersuchung handelt es sich bei der vorliegenden Arbeit.

Verhängnisvoll bei der Bearbeitung der Barockliteratur war eine gewisse stilistische Schwäche: Herbert Cysarz, Günther Müller und leider auch Paul Hankamer, (denen wir im übrigen viel verdanken), glauben die Einfühlung in die barocke Lebensform so weit treiben zu müssen, dasz sie ihre Sprache annehmen. Martin Sommerfeld äussert sich in seinem Artikel "The 'Baroque' Epoch in German Literature," (1939), über diese Kalamität in folgender Weise: „I felt more annoyed than assisted in my own course, by the hasty generalizations and not the least, by the expressionistic style in which the three books (s. Anm. 3) on Baroque literature were composed." 2 Man wird in solch einem Verfahren für Liebhaber keine Gefahr sehen; doch gibt es auch heute noch Literarkritiker, die dem „Barockproblem” verständnislos gegenüberstehen, und vom Nicht-Verstehen ist es nur ein kleiner Schritt zur Opposition (er ist menschlich!). In diesem Falle wird eine solche stilistische Schwäche zur „Waffe in der Hand des Gegners," was nicht 
so pathetisch gemeint ist, wie es sich anhört, und letzten Endes nur eine Situation kennzeichnet. ${ }^{3}$

Der Autor einer Studie über Barockliteratur hat ferner zu entscheiden, ob er sich dem Thema auf generellem Wege (deduktiv) oder in eingehender Einzelbeschreibung (induktiv) nähern will. Es wäre eine grosze Hilfe gewesen, hätte Paul Hankamer seiner Behandlung des Barock $a b$ und $z u$ einmal einige Originaltexte der betreffenden Autoren beigefügt; seine Darstellung hätte ungemein an Interesse und Wert gewonnen. Im Gegensatz dazu ist Frickes Buch über Gryphius zwar wertvoll, aber es verliert sich ein wenig in den Einzelheiten und verfällt ins andere Extrem. "Definition des Begriffes 'Barock" ist ein „,unglückliches" Thema (jedes Thema hat seine Eigengesetzlichkeit und kann nicht in jeder Weise behandelt werden): anerkannterweise ist der Begriff „Barock” schwer bestimmbar, Widersprüche werden sich kaum vermeiden lassen. Auszerdem ist Welleks Artikel zu lang (im Verhältnis zu seiner Fragestellung) und fällt etwas auseinander, auch unterscheidet der Autor nicht genügend zwischen ,,volkstümlichen" Interpretationen des Barock und den mehr wissenschaftlichen Definitionen. Freilich ist uns Welleks umfangreiche Bibliographie hochwillkommen, was auch von der des Hans Pyritz in Hankamers Buch gesagt werden kann. Über Croce und die Gefahren der neoklassischen und positivistischen Kritik braucht hier nicht mehr gesprochen zu werden ${ }^{4}$. Dagegen stellt Jean Roussets neues Buch, La Littérature de l'âge baroque en France (Paris, 1953) einen recht wertvollen Beitrag zur Barockforschung dar.

ii. Barocke Thematik in der Lyrik des Andreas Gryphius.

Nach eingehendem Studium der vorhandenen Sekundärliteratur hat sich die Verfasserin der vorliegenden Arbeit entschlossen, zwar auf induktivem Wege vorzugehen, aber im Hinblick auf den verhältnismäszig geringen Umfang dieser Studie ein eklektisches Prinzip walten $\mathrm{zu}$ lassen: wir werden unser Augenmerk nur auf Hauptthemen in der Lyrik des Gryphius richten und alle Ausnahmeerscheinungen beiseitelassen. Die Gelegenheitsdichtung, die zugegebenerweise einen groszen Teil seines lyrischen Schaffens ausmacht, wird kaum berührt werden. Durch gelegentliche Seitenblicke auf Gryphs dichterische Zeitgenossen nicht nur in Deutschland, sondern auch in den umliegenden Ländern, soll gezeigt werden, in welchem Masze Themen der deutschen Barockdichtung im Einklang stehen mit allgemein europäischen Fragestellungen im siebzehnten Jahrhundert. Denn Barock ist weder ein nordisches Gewächs, noch eine Begleiterscheinung der Gegenreformation (und nichts anderes), weder rein protestantisch noch katholisch, weder spanisch, noch italienisch, noch deutsch; Barock ist in dem gleichen Sinne europäisch, wie zum Beispiel Lateinisch für lange Zeit die europäische Gelehrtensprache gewesen ist, oder wie 
beinahe bis auf den heutigen Tag Französisch die allgemein anerkannte Diplomatensprache ist. Dasz wir modernen Menschen vergessen haben, wie man in groszen Konzeptionen denkt, ist einer Entwicklung zuzuschreiben, die in dem Aufkommen der Nationalstaaten ihren Anfang genommen hat. Wenn wir uns nicht beeilen, wird uns die neuere politische Entwicklung vor eine Entscheidung stellen und uns vielleicht zu einem solchen Denken zwingen.

\section{a. Im Wirbel der Ereignisse.}

Wenn auch Grimmelshausens Simplicissimus die erste Quelle für das Weltbild des siebzehnten Jahrh underts ist, so gibt uns doch Gryphius in seiner Lyrik nicht weniger eindrucksvolle Skizzen:

Wir sind doch nunmehr gantz, ja mehr denn gantz verheeret.

Der frechen völcker schaar, die rasende posaun,

Das vom blut fette schwerdt, die donnernde carthaun

Hat aller schweisz und fleisz und vorrath auffgezehret.

Die thürme stehn in 'glut, die kirch ist umgekehret,

Das rathhaus liegt im graus, die starcken sind zerhaun,

Die jungfern sind geschänd't, und wo wir hin nur schaun,

Ist feuer, pest und tod, der hertz und geist durchfähret.

(S. I I 3).

Was war der Dreiszigjährige Krieg anderes als das sichtbare Zeichen für den Untergang einer alten Welt! Die mit dem „Herbst des Mittelalters" einsetzende Renaissance und Reformation hatten--jede in ihrem Gebiet--zu eruptiv gewirkt, als dasz der nunmehr seelisch und geistig emanzipierte Mensch sich in der neuen Welt hätte zurechtfinden können. Noch war der absolutistische Staat nicht geboren, noch gab es weder geordnete Wirtschaftssysteme, noch stehende Heere; und diese Welt stellte bald mehr Anforderungen, als dasz ihnen ein Mensch der Renaissance gewachsen sein könnte. Noch weisz man wenig über die Kraft fanatischer Massen, steht man verständnislos der Zündkraft gewisser Interpretationen der Bibel gegenüber; verstehende Gelehrte sehen sich gezwungen, Skeptiker oder Nihilisten zu werden, und ziehen sich vom Schauplatz zurück. Damit ist aber dem Volk nicht geholfen. Es steht vor den Trümmern seiner Weltordnung: „Die thürme stehn in glut, die kirch ist umgekehret"--und ist geneigt, den Ereignissen kosmische Bedeutung zuzuschreiben. Man glaubt an ein verhängtes Schicksal, man kann die Ursache nicht sehen. Eine Weile lang hatten diese Menschen geglaubt, sie könnten die Welt bezwingen: neue Erdteile wurden entdeckt, neue Gebrauchsgegenstände erfunden, neue Wege der Mitteilung und Wissensverbreitung geebnet. Doch langsam beginnt sich der heitere Renaissancehimmel zu trüben. Wie kann man der Pest beikommen? oder: Was nutzen mir meine Bücher und Kleinodien, wenn ich alt werde und sterbe? Das waren Fragen, 
die sich der Mensch der Frühbarockzeit wohl stellte, zusammen mit anderen, schwereren, wie zum Beispiel: Wir, die Protestanten, beten zu Gott; drüben die Katholiken fünfzig Meilen entfernt von hier beten zu dem gleichen Schöpfer--wer von uns ist rechtgläubig? Man kommt wohl auch zu der groszen Frage, die sich nach der kopernikanischen Entdeckung erhob: Wenn die Erde nicht der Mittelpunkt des Universums ist, wie ist dann unsere Stellung als Menschen zu Gott? Noch gab es keinen Leibniz, der diese Menschen mit seiner Lösung getröstet und ihnen neue Sicherheit gegeben hätte. Eines scheint dem Barockmenschen schmerzhaft klar zu werden: er kommt mit all seinem Wissen und den Errungenschaften seiner Zeit nicht weiter. Gryphius sagt einmal: „Ists? oder wissen wir weit minder, als man sieht?" (S. I 40). Irgend etwas ist nicht in Ordnung. Und die grosze Unbekannte, die der Menschen Berechnungen im Handumdrehen unwirksam macht, wächst (wie schon oben angedeutet) ins Kosmische im gleichen Verhältnis, wie das Vertrauen in die eigene Kraft vormals stark gewesen war:

Der dicke querbaum bricht und schlägt den umgang ein;

Das seege' flattert fort; der schiffer steht allein

Und kan noch boots-mann mehr, noch seil, noch ruder zwingen.

(S. 174).

Hier wie im folgenden $\mathrm{G}=\mathrm{Ji}^{\mathrm{i}} \mathrm{cht}$ bedient sich Gryphius der Natursymbolik, indem er solche Naturphänomene wählt, die im Gegensatz zum gewöhnlichen Ablaur der natürlichen Erscheinungen stehen:

Offt, wenn uns schwartze nacht im mittag überfiel, Hat der geschwinde blitz die seegel schier verbrennet.

Wie offt hab ich den wind und nord' und sud verkennet!

Wie schadhafft ist der mast, steur, ruder, schwerdt und kiel!

(S. 126$)$.

Selbst die Hölle wird durch Natursymbolik charakterisiert:

Ach vergeh / Tieff und höh'!

Meer! Hügel! berge! fels! wer kan die pein ertragen!

Schluck abgrund! ach schluck ein, die nichts denn ewig klagen! Je und eh!

(S. 156$)$.

Kennzeichnend ist schon hier, bei Gedichten, die in allgemeiner Weise 'seine Zeit veranschaulichen, die direkte Verbindung zu Gott in der jeweils letzten Strophe, in Form einer geradlinigen Transzendenz; so schlieszt Gryphius das Gedicht Andencken eines auf der see ausgestandenen gefährlichen sturms (an der Küste von Rügen, im Juni I638) mit: 
Wir missen glas, compasz und tag und stern und nacht;

Todt war ich vor dem todt. Doch herr! du hasts gemacht,

Dasz ich dir lebend und errettet lob kan singen.

(S. I74).

Wo Gryphius sich nicht Gott selbst zuwendet, findet die Transzendenz der letzten Strophe in Form eines Überganges ins Innere, Seelische, statt, wie im letzten Terzett des Sonettes Thränen des vaterlandes, anno 1636 :

Doch schweig ich noch von dem, was ärger als der tod, Was grimmer denn die pest und glut und hungersnoth.

Dasz auch der seelen-schatz so vielen abgezwungen.

\section{b. Eschatologisches.}

Ereignisse, die ins Menschenleben eingreifen und in Folge ihrer Unerklärbarkeit ins Kosmische wachsen, werden bald in engen $\mathrm{Zu}$ sammenhang mit dem Jüngsten Gericht und allgemeiner eschatologischer Thematik gebracht. Wie im ausgehenden Mittelalter wenden sich viele Maler im Barockzeitalter wieder der Motivik des Jüngsten Gerichtes zu und bereichern die Vorstellungswelt ihrer Zeitgenossen durch Ausmalung des Geschickes der abgeschiedenen Seelen (Hermann tom Ring; Rubens). Auch aus Eintragungen in die Kirchenbücher sowie aus der Literatur erhalten wir mehr als einen Beweis, dasz die Menschen jener Zeit unter dem Eindruck der katastrophalen Ereignisse tatsächlich glaubten, der Jüngste Tag sei angebrochen. Durch das ganze Jahrhundert rechnet man mit der Wiederkunft Christi und dem Anbruch seines Reiches; die Geschehnisse der Zeit werden prophetisch ausgedeutet ${ }^{5}$. So schreibt Gryphius in den Dissertationes funebres, S. 576: „Wer wil zweiffeln, dasz anietzt die letzten Tage! Wir sehen den Greuel der Verwüstung, unser Erlöser heist uns fliehen! wo aber hin? der gantze Creisz der Erden lodert in dem Feuer des Zornes Gottes ...."6. In seinem Gedicht Auf den tag Matthäi heiszt es dann:

Ach eile! reisz dich los! itzt kommt der heyland an!

Itzt rufft und sucht dich der, so alle wil befreyen,

Der uns von schuld entbindt, der willig zu verzeihen,

Der auch, was kranck und todt, bald lebend machen kan.

(S. 88).

Sieghaft und majestätisch erklingt das gleiche Thema in den vierfüszigen Jamben der Dichtung Gedanken über den kirch-hof und ruhestädte der verstorbenen: 
Herr! wenn mein geist nur stehen kan

Und ich vor deinem richtstuhl siege.

Ich weisz, die angesetzte zeit

Wird bald mit ungeheuren krachen

Und lichter glut das vorspiel machen

Der unbegräntzten ewigkeit.

Wenn Gottes letztes feldgeschrey

Verstärckt mit blitzen und trompeten,

Wird durch der langen länder rey

Erschallen und den tod ertödten,

Wenn marmor, ertz, metall und stein

Und Pharos unterirrd'sche grüffte

Vor lieffern werden in die lüffte

Die leichten geister-vollen bein.

(S. 349-350).

\section{c. Die Zeit.}

Im ersten Teil des obigen Zitates stehen wir zwei Begriffen gegenüber, die in ihrer Antithetik nicht nur das Werk des Gryphius beherrschen, sondern auch kennzeichnend für die gesamte Barockliteratur sind. (Ich bin mir der Tragweite einer derartig superlativischen Behauptung durchaus bewuszt, glaube sie aber in jedem einzelnen Fall rechtfertigen zu können). Es sind die Begriffe "die angesetzte zeit" und die ,unbegräntzte ewigkeit." In diesem so eigenartigen siebzehnten Jahrhundert beschäftigt man sich gedanklich mit der Zeit in einer Intensität, die ein moderner Denker gern als pathologisch bezeichnen würde. Fritz Strich schreibt 1947: „Die Zeit: sie ist die unheimliche Göttin des Barock "'. Zum ersten Mal in der Geschichte der Menschheit, soweit wir sie zurückverfolgen können, werden sich die Menschen schmerzhaft ihres (metaphysischen) Seins in der Zeit bewuszt, in einem Gefühl äuszerster Erschütterung, das in seiner Ausweglosigkeit bei weitem den Nachhall der Vanitas-Idee der Kirchenväter übersteigt. Die Kluft zwischen Gott und Menschheit ist weit aufgerissen, so weit, dasz eine Neuvereinigung unmöglich erscheint; wo sie dennoch geschieht, hat man den Eindruck, dasz die Menschen sich im letzten Augenblick an diese Gottesidee klammern, in der dunklen Ahnung, dasz der Gottesbegriff gefährdet ist. Bei Gryphius gelingt die Transzendenz in direkter, wenn auch antithetischer, Form, und sie erinnert ein wenig an die Gotteslehre des Nicolaus von Cusa, nach der (in abgekürzter Form) Gott all das ist, was die Menschen nicht sind. Ein Jahrhundert später wird die Gefahr für den Gottesbegriff akut.--Im modernen Existentialismus ist die Problematik "Sein und Zeit" wiederaufgenommen worden und hat durch Heidegger einerseits und Jaspers von seiten der Theisten eine Um- und Neuwertung erfahren.

Diese Zeit, das Attribut irdischen Lebens, wird häufig in der Barock- 
literatur personifiziert (wie am Ende der dritten „Abhandlung" von Cardenio und Celinde), und sie steht fast immer dem Menschen als Feindin gegenüber, die in sein Leben eingreift:

Mein sind die jahre nicht, die mir die zeit genommen;

Mein sind die jahre nicht, die etwa möchten kommen.

„Der Augenblick ist mein,” fährt Gryphius fort, aber nicht, um diesen zu genieszen, wie alle Renaissancedichter in Nachahmung der antiken Hedoniker ein solches Epigramm zu Ende geführt hätten, sondern im Sinne stoischer Vorbereitung auf den Tod:

Der augenblick ist mein, und nehm' ich den in acht,

So ist der mein, der jahr und ewigkeit gemacht.

Der Stoizismus des Barocks ist eine der Hauptkomponenten im Ideengut des siebzehnten Jahrhunderts. Man sucht Seneca hervor, der in der Renaissance ein wenig in Vergessenheit geraten war, und vertieft die stoischen Lehren der Absagung an das Irdische, der Vorbereitung für ein künftiges Sein, von dem man !nicht mehr weisz, als dasz es in allen Punkten anders ist als das augenblickliche Sein. In schritthafter, exerzitienartiger, methodischer Vorbereitung stellt man sich auf das Unvermeidliche ein. Es ist von Wichtigkeit, dasz wir uns die Grundprinzipien der stoischen Haltung und die fast heitere Ruhe ihrer Unerschütterlichkeit (Atharaxie) klar vor Augen stellen, schon um zu erkennen, dasz Andreas Gryphius durchaus nicht immer stoisch denkt. Zwar ist er mit dem Neo-Stoizismus in Holland in engen Kontakt gekommen, und ein Gedicht wie (die oben erwähnte) Betrachtung der Zeit läszt darüber keinen Zweifel zu, ebenso wenig wie das Ende des Trauerspiels Cardenio und Celinde (,,Wer hir recht leben will und jene Kron ererben / Die uns das Leben gibt; denck jede Stund ans Sterben."). In den Niederlanden war Senecas Philosophie schon um i60o durch Männer wie J. Lipsius und C. Scioppius, Vossius und Heinsius, im christlichen Sinne ausgedeutet und waren stoische Ideen in das protestantische Christentum geleitet worden. Andererseits aber zeugen Worte wie „Wenn, was die Zeit siebt in die Luft," oder „dies Leben fleucht davon wie ein Geschwätz und Scherzen," und "dies Leben kömmt mir vor als eine Rennebahn" in Gryphs Lyrik, wie auch jene Verse: „die Zeit, die auf mich Angst und grimmer Seuchen Schar / Und Trauren und Verdrusz und Schrecken hat verhetzet"---von einer ganz unstoischen Erschütterung, die sich aus einem Grund des inneren Widerstrebens, einer heimlichen Lust am Leben, erhebt. Walther Rehm faszt diesen Gedanken in folgender Weise: „Die Zeit wird in das Jenseitige aus selbstquälerischem Zwang hineingetrieben, weil (Rehm unterstreicht) sie so am Leben hängt, und doch wieder aus dem Erlösungswillen, aus der Angst vor dem Leben und dem Gefühl, 
dasz plötzlich der Boden unter den Füszen schwindet. Es ist ein verzweifeltes Sichanklammern und Haltsuchen ${ }^{8}$." Ermatinger ${ }^{9}$ sagt in ähnlichem Zusammenhang: „Die Idee der Askese ist nur der eine Pol in dem Kräftespiel jenes Zeitalters." Als Gegenpol findet sich „ein zweites Kräftezentrum, das seinen Grund hat in dem natürlichen Wesen des Menschen, der Begehrlichkeit seiner Sinne, der Stärke und Gesundheit seines Körpers, der Lockkraft dessen, was schon den Menschen des Mittelalters als die „Welt" anzog."

Gryphius ist weder lebensmüde noch pessimistisch, wir können Schieck und Fricke nicht zustimmen ${ }^{10}$. Der Stoiker hat die Zuversicht, mit dem Leben fertig zu werden; Gryphius hat sie nicht. So grauenvoll es oft auch erscheint--es ist doch verlockend. „Ihr bücher, meine lust. . Ihr Musen, meine wonne" ruft er in der Ode Dimitte me, ut plangam paulum dolorem meum (S. 274), voller Schmerz, dasz er sie lassen soll. Solch eine weltbejahende Haltung findet ihren Höhepunkt in Auf seinen geburtstag:

Danck sey dir! ewig danck, vor höchste wunderthaten!

Danck sey dir, herr! dasz ich dein wunderwerck an mir

Und allmacht deiner hand und höchste gunst verspür,

Dadurch in mutterleib ich dein geschöpff gerathen!

Du hast mit deinem licht in den verborgnen schatten

Die seel in mir entsteckt; die glieder, die ich führ,

Der beiner meisterstück, das fleisch, der adern zier sind wunder.

(S. 172).

Im ganzen gesehen wirft Gryphius die Problematik auf--und läszt sie offen; seine Dichtung wirkt daher als unmittelbares Spiegelbild jener Dualität, die wir oben als kennzeichnend für das Barock hingestellt hatten: Menschheit--Gott, Zeit-Ewigkeit, Diesseits-Jenseits. Solch direkte Antithetik erscheint in den Worten: „Er lebt, indem er stirbt; er steigt, indem er fällt," und nicht zuletzt im letzten Terzett des vielzitierten Einsamkeitssonettes:

Der mauren alter graus, disz ungebaute land Ist schön und fruchtbar mir, der eigentlich erkannt, Dasz alles, ohn ein geist, den Gott selbst hält, musz wanken. (S. 133).

Die Antithetik des Gedichtes An den heiligen Geist (S. I66) ist die Sprache der Mystiker; je weiter man in der Betrachtung der Gryphschen Lyrik vordringt, desto mannigfaltiger werden die formalen Eindrücke. Gryphius läszt sich schwer klassifizieren; ein einseitiges Urteil, das ihn zum Mystiker erklärt, ist ebenso unrichtig wie eine zu starke $\mathrm{Be}$ tonung des stoischen Charakters dieser Lyrik, denn in gewissem Sinne ist sie alles dies zu gleicher Zeit. Wir dürfen nicht vergessen, dasz Gryphius-in Ermanglung eines besseren-zu traditionellen Sprach- 
formen greift und mittelalterliche Elemente und Renaissance-Formen eklektisch verwendet, wo es ihm geraten erscheint, sie aber sprengt, wenn sie der Emphase seines dichterischen Ausdrucks nicht mehr gewachsen sind. Ein gutes Beispiel für die Sprengung des Sonetts (einer Schöpfung der Renaissance) ist das Gedicht Die hölle, dessen zweites Quartett ich weiter oben zitierte. Hier ist das erste:

Ach und weh!

Mord! zetter! jammer! angst! creutz! marter! würme! plagen!

Pech! folter! hencker! flamm! stanck! geister! kälte! zagen!

Ach vergeh ....

\section{d. Vanitas.}

Das antithetische Element in der Lyrik des Gryphius verdichtet sich wohl am stärksten in den Versen, die dem Thema "Vanitas" an sich gewidmet sind. Ich denke dabei an Worte wie die ,"tolle Herrlichkeit der Welt," das „prächtig aufgeschmückte Nichts," an Verse wie:

Ich der ich asch und kot, mag ich mich unterfangen,

Den Sünd und fluch beschwert, zu knieen, Herr, vor dich?

und dann vor allem an das Sonett Einsamkeit, die Ode Vanitas! Vanitatum Vanitas!, Verläugnung der Welt, Es ist alles eitel und Überschrift an dem Tempel der Sterblichkeit ${ }^{11}$. Auch der ,,abenteuerliche Simplicius Simplicissimus" war zu dem Schlusz gekommen, " dasz nichts Beständigers in der Welt ist, als die Unbeständigkeit selbsten"; gegen Ende dieses ersten deutschen Romans kehrt das gleiche Thema wieder:

O wunderbares Tun, o unbeständig's Stehen!

Wann Einer wähnt, er steht, so musz er fürdergehen.

O schlüpferigster Stand!

Wegen dieser geistigen Verwandtschaft stellt Walther Rehm Gryphius und Grimmelshausen heraus ,,als die Gipfel und Grenzen des deutschen Barockjahrhunderts; sie drücken als besonders Ragende aus, was alle andern auch bewegt" (s. Anm. 8, S. I89-190), und sind, nach Erich Trunz, ,,beinahe zu einer 'inneren Form' im Sinne Humboldts gelangt ${ }^{12}$."

Auch in den umliegenden Landern herrscht die Vergänglichkeitsthematik und der Gedanke von der Entwertung der „Welt." Dem Grimmelshausen ebenbürtig ist Cervantes. Hauptmotiv des Don Quijote ist die Problematik der Wirklichkeit, auf Grund deren das Leben zwischen Schein und Wahrheit zu oscillieren scheint. Die Menschen dieses Romans (sowie anderer Werke des groszen Spaniers) sind Suchen- 
de, Wanderer, die ihr Ziel zeitweise aus den Augen verlieren. Ihr Weg führt durch Irrtum und Miszverständnis, ihr Handeln wird einer Kritik unterzogen, die von auszerhalb der Gesellschaft stehenden Elementen (von Geistesgestörten, Zigeunern, Vagabunden, und-Tieren) ausgeht. Manche dieser Menschen irren ihr Leben lang (Persiles, Don Quijote), um erst im Augenblick des Todes zur Erkenntnis zu kommen.

In der gleichen Tonalität steht Calderons Werk:

Was ist Leben? Raserei!

Was ist Leben? Hohler Schaum,

Ein Gedicht, ein Schatten kaum!

Wenig kann das Glück uns geben,

Denn ein Traum ist alles Leben

Und die Träume selbst ein Traum.

Shakespeare hatte die Welt mit einer Bühne verglichen (As You Like It, II, 7):

All the world's a stage,

And all the men and women merely players:

They have their exits and their entrances.

Der Gedanke der Vanitas ist die Reaktion des Menschen dieses Jahrhunderts auf die plötzliche Erkenntnis seines Seins in der Zeit. Die Frage nach der Rangstellung des Menschen ist alt; in der Antike hatte man sich bereits um die Begründung der "dignitas hominis" bemüht. In der gleichen Absicht versuchte die mittelalterliche Patristik, Griechisches (in ciceronianischer Gestalt) und Biblisches miteinander zu verschmelzen. Cicero hatte platonisch-aristotelische Bestimmungen der menschlichen Wesenswürde (,,humanitas") neu formuliert, die besagten, dasz der Mensch ein Mischgebilde aus tierischer und geistiger Vernunft ist, mit der Gabe, die letztere von jener zu lösen und bis zur Gottesnähe zu steigern. Diese Charakterisierung liesz sich nun gut auf biblisch-theologische Motive übertragen, wie: der Mensch ist die Krone der Schöpfung, das Ebenbild Gottes ${ }^{13}$.

Neben die Dignitas-Thematik (nicht in den Gegensatz zu ihr) stellte die mittelalterliche katholische Theologie eine zweite Themengruppe: "miseria hominis." Sie bringt die Unzulänglichkeit und Todesverfallenheit des Menschen zum Ausdruck, stellt aber (im Mittelalter noch) die Gottesebenbildlichkeit und Begnadbarkeit seines Wesens nicht in Zweifel. In der Renaissance verblaszt die Miseria-Thematik zugunsten des Würdegedankens, der sich nun in den erwachenden Naturwissenschaften neu begründet.

Doch schon in der Spätrenaissance, in der zweiten Hälfte des sechzehnten und um die Wende zum siebzehnten Jahrhundert, als die 
Ereignisse die Menschen eine Krisis ahnen lieszen, flackerte die MiseriaIdee wieder auf. Man kann diese Entwicklung gut in kulturkritischen Schriften verfolgen, ich denke hier besonders an Montaigne. Der Gedanke von der Machtlosigkeit des Menschen wird in solchen Schriften gern kombiniert mit einer Kritik der zivilisierten Welt, im Hinblick auf die weniger entwickelten, aber dafür unverdorbenen ethnischen Gruppen entfernter Weltteile, die den zersetzenden Kräften des Geistes weniger ausgesetzt, doch den animalischen Quellen ihres Seins näher sind. Im Gegensatz zum ciceronianischen ,,praestare bestiis" von einst benutzt man die Tierallegorik, um dem Menschen die Begrenztheit seiner „,conditio humana" vor Augen zu stellen. Die Narrenliteratur ist (im philosophischen Sinne) nichts anderes als die Veranschaulichung einer Erkenntniskritik, ein Zweifeln daran, dasz der menschlichen Vernunft keine Grenzen gesetzt sind. Zusammen mit den sichtbaren Zeichen für des Menschen Vergänglichkeit formen solche Gedanken die Barockausprägung der Vanitas- und Miseria-Idee, der wir in den oben erwähnten Gedichten des Andreas Gryphius gegenüberstehen.

Interessant ist das Sonett Einsamkeit. Es erinnert an romantische Dichtungen und ist doch zu gleicher Zeit typisch für den Wandel, der sich zwischen Spätrenaissance und Hochbarock vollzogen hat, Sinnende Menschen des sechzehnten Jahrhunderts grübelten in ihrem Studierzimmer über die Vergänglichkeit der Welt (Dürers Hieronymus; Faust), in enger Nachbarschaft mit Stundenglas und Totenschädel; Gryphius, der grosze Einsame, geht hinaus in die Natur:

In dieser einsamkeit der mehr denn öden wüsten, Gestreckt auf wildes kraut, an die bemooste see,

Beschau ich jenes thal und dieser felsen höh',

Auf welchen eulen nur und stille vögel nisten.

Hier, fern von dem pallast, weit von des pöbels lüsten,

Betracht ich, wiz der mensch in eitelkeit vergeh',

Wie auf nicht festem grund' all unser hoffen steh',

Wie die vor abend schmähn, die vor dem tag uns grüszten. (S. 133).

In der Vanitas-Ode heiszt es dann:

Die herrligkeit der erden / Musz rauch und aschen werden, Kein fels, kein ertzt kan stehn.

Disz was uns kan ergetzen, / Was wir für ewig schätzen, Wird als ein leichter traum vergehn.

Die Gleichsetzung des Lebens mit dem Traum erscheint häufig in der Literatur des Barock, um damit die Nichtigkeit des Lebens zu charakterisieren (Calderón, s.o.); andererseits verwendet man aber auch den Traum als Kategorie des Phantastischen in kulturkritischer Absicht, um darin ein besseres, ethisch reineres Leben auszumalen. 
Der ruhm, nach dem wir trachten, / Den wir unsterbiich achten, Ist nur ein falscher wahn.

So bald der geist gewichen / Und dieser mund erblichen, Fragt keiner, was man hier gethan.

Wie deutlich zeichnet sich in diesen Versen die Entwicklung ab, die die Ruhm-Idee der Renaissance erfahren hat! Noch bei Weckherlin wurde "gloria" anders behandelt:

Ein solcher Tod (d. h. Schlachtentod) ist ihm nicht schwer, Weil sein Gewissen ihn versüszet

Und er erwirbet lob und ehr

Indem er sein blut so vergieszet ${ }^{14}$.

In der folgenden Strophe der Ode erfolgt eine Neuverwertung der lyrischen Metaphorik des Ausonius, im elegischen Rosen-Motiv, das aber, im Gegensatz zur Renaissance, von einer epikuräischen Lösung absieht, die empfohlen hätte, den ach nur so flüchtigen Augenblick zu genieszen:

Wie eine rose blühet, / Wenn man die sonne sihet

Begrüszen diese welt,

Die eh der tag sich neiget, / Eh sich der abend zeiget,

Verwelckt und unversehns abfällt.

Die Stimmung dieser Ode ist ernst, aber nicht verzweifelt; tiefer und hoffnungsloser klingen die Sonette Es ist alles eitel und Menschliches Elend (S. I03), während die stärkste Pathetik wohl in Verläugnung der Welt zu finden ist. Die beiden Sonette sind eng miteinander verwandt, und nicht umsonst stellt sie Hederer in seiner neuen Anthologie zusammen ${ }^{15}$. In beiden werden die Themen Eitelkeit und Vergänglichkeit ausgearbeitet, jeweils im ersten Terzett erfolgt die Gleichsetzung des Lebens mit dem Traum und die Herabsetzung des Ruhmes- und Unsterblichkeitsgedankens; und doch ist die Stimmung des zweiten Sonettes eine andere, über einem Grundton schmerzhaften Begreifens:

Was sind wir menschen doch! ein wohnhaus grimmer schmertzen, Ein ball des falschen glücks, ein irrlicht dieser zeit,

Ein schauplatz herber angst, besetzt mit scharffem leid, Ein bald verschmeltzter schnee und abgebrannte kertzen.

Im letzten Terzett erscheint eine Reminiszenz an den alten Spruch der Legende von den drei Lebenden und den drei Toten (,Was ihr seid, das waren wir / Was wir sind, das werdet ihr" ${ }^{16}$ ): 
Was itzund athem holt, musz mit der lufft entfliehn,

Was nach uns kommen wird, wird uns ins grab nachziehn.

Was sag ich? wir vergehn, wie rauch von starcken winden.

(S. 104).

Hier möchte man einem Vergleich mit Christian Günthers Die Eitelkeit des menschlichen Lebens Raum geben, um festzustellen, wie verschieden beide Dichter dieses Thema behandeln; Günther spricht in der folgenden Strophe vom Tod:

Drauf fährt man nackt und blosz / Nach einem finstern Lande Auf die Verwesung los;

Ja mancher musz sein Sterben, / Ach Ungemach!

Noch wohl mit Angst erwerben. / Ach! Ach!

Hofmannswaldau andererseits spart nicht mit Antithetik, zum Beispiel in Die Welt; doch glaubt man auch bei ihm nicht die starke seelische Erschütterung wahrzunehmen, die das schöpferische Element in Gryphius bildet.

Wie im Sonett Die hölle (s.o.), so auch in Verläugnung der Welt sprengt die Dynamik des Gehaltes die äuszere Form in typisch barocker Weise, und nebeneinandergesetzt, -gehäuft, werden Substantiva, wie die unförmigen Marmorblöcke einer zerbrochenen Renaissance-Säule:

Verhüllen mein gemüth, und alle phantasie

Der eitel-leeren welt sey für mir als ein traum,

Von dem ich nun erwacht! Und lasz nach diesem tod,

Wenn hin dunst, phantasie, traum, tod, mich ewig stehn.

(S. 237).

Wie mit der Schönheit der leblosen Welt verfährt Gryphius auch mit menschlicher Schönheit: er sieht sie nicht als solche, sondern stets als schon dem Verfall anheimgegeben, als schon die Keime der Fäulnis in sich tragend. Cardenio folgt einer schönen Frauengestalt-da heiszt es in den szenischen Erklärungen: „Der Schau-Platz verändert sich plötzlich in eine abscheuliche Einöde / Olympie selbst in ein Todtengerippe / welches mit Pfeil und Bogen auff den Cardenio zilet." Höchste Schönheit verwandelt sich urplötzlich in gräszliche Verwesung, eine Antithese, die schon bei Spangenberg auftritt ${ }^{17}$. An die Stimmung des Sonetts Über die gebeine der ausgegrabenen Philosetten erinnern die Worte, die der "Mensch" (in: Cardenio und Celinde, 3. Abh.) beim Anblick der Alten ausstöszt, die den Winter verkörpert:

$O$ häszlich Frauen-Bild! was ist die Fackel noth!

Bist du mir in mein Grab zu leuchten vorgegangen!

$O$ lebend Sichen-Hausz / O Muster von dem Tod! 
Für die (sehr interessante) Alterstypologie in Gryphius dürften im übrigen die Leichenreden recht ertragreich $\operatorname{sein}^{18}$.

Eine ausgesprochen erkenntniskritische Note, die an die Bedeutung des "Irrtums" in Cervantes erinnert, trägt das Sonett Überschrift an dem tempel der sterbligkeit (S. I35); zugleich läszt sich an ihm gut die antithetische Form studieren:

Ihr irrt, indem ihr lebt; die gantz verschränckte bahn Läszt keinen richtig gehn, Disz, was ihr wünscht zu finden, Ist irrthum, irrthum ists, der euch den sinn kan binden.

Was euer hertz ansteckt, ist nur ein falscher wahn.

Im ersten Terzett verstärkt sich die antithetische Verschränkung:

Ihr irrt, indem ihr schlafft; ihr irrt, indem ihr wachet;

Ihr irrt, indem ihr traurt; ihr irrt, indem ihr lachet;

Indem ihr disz verhöhnt und das für köstlich acht.

Hier mag man an einige Verse aus Cardenio und Celinde denken, in denen Olympia ihre Lage einem Irrtum von seiten Cardenios zuschreibt:

Was biszher je von ihm / zu wider mir geschehn;

Rührt daher / dasz er mich nicht selbst hat angesehn.

Ihn hat mein nichtig Fleisch / der falsche Schnee der Wangen

Und des Gesichtes Larv / und diser Schmuck gefangen

Den mir die Zeit abnimmt; nun hat die wahre Nacht

Mein Antlitz recht entdeckt. Herr! diser Lilien Pracht

Des Halses Elffenbein sind nur geborgte Sachen.

e. Der Tod.

Die Erfahrung der Vanitas begründet sich im Erlebnis des Todes. Abgesehen von allgemeinen Eindrücken aus der Atmosphäre des Dreiszigjährigen Krieges wird uns berichtet, dasz Gryphius beide Eltern früh verlor, und es scheint, dasz auch seine Freunde ihm jeweils in dem Augenblick entrissen wurden, als er ihrer am meisten bedurfte. So schreibt er später:

In meiner ersten blüt', im frühling zarter tage

Hat mich der grimme tod verweiset und die nacht

Der traurigkeit umhüllt,

oder in Auf die letzte nacht seines XXV. Jahres:

Komm mitternacht und schleusz disz thränen-reiche jahr,

Die schmertzen-volle zeit, die mich so tieff verletzet,

Die dich, mein bruder! hat in jenes reich versetzet,

Und schwester! deine leich gestellet auf die baar.

(S. 138 ). 
Unter seinen Gelegenheitsgedichten sind wohl die Begräbnislieder und Nachrufe am zahlreichsten. Der Todesgedanke beherrscht Gryphius in einem Masze, das in seiner Ungewöhnlichkeit bewirkt, dasz eigentlich nur die Menschen ihn voll verstehen werden, die Ähnliches wie er erlebt haben. Krankheit am eigenen Körper wird sofort mit der Todesidee verbunden und als allmähliches Absterben oder Verfaulen des Körpers erkannt. Prototypisch für solche Thematik ist das Sonett An sich selbst (ein echtes Anthologie-Stück):

Mir grauet vor mir selbst; mir zittern alle glieder,

Mein cörper ist nicht mehr als adern, fell, und bein.

Das sitzen ist mein tod, das liegen meine pein.

Die schenckel haben selbst nun träger wohl vonnöthen.

(S. 125$)^{19}$

Hier bleibt uns weitere Ausführung erspart; es ist charakteristisch, dasz Gryphs ausdrucksvollste Gedichte „sich selbst kommentieren" (es ist in dieser Hinsicht leider von den Verfassern monographischer Studien über Gryphius des Guten zuviel getan worden). Man vergleiche aber vielleicht mit diesem Sonett das rein stoische An sich von Paul Fleming: welch ein Unterschied in Dimension!

Den wohl realistischsten Ausdruck in Gryphs Lyrik tragen die Gedancken über den kirch-hof und ruhe-städte der verstorbenen (S. $340 \mathrm{ff}$.). Hier, wie es uns scheint, flieszt in gedrängter Form alles das zusammen, was mehrere Jahrhunderte an Vorstellungskraft aufbringen konnten, um in realistischer Schilderung jenen Zustand auszumalen, in dem jeder, ob arm oder reich, gelehrt oder unwissend, sich einmal befinden wird. Wir denken beim Lesen dieser Zeilen an die Verse, die die alten Totentanzdarstellungen begleiteten, wir bringen die vor uns aufsteigenden Bilder in Verbindung mit den Illustrationen dieser Tänze. Vom Vergleich des Kirchhofes mit einem Garten, (der im siebzehnten Jahrhundert noch gegensätzlich ausfallen muszte) geht Gryphius dazu über, ihn als Schule im stoischen Sinne zu betrachten. Der Friedhof wird zur Schule des Verlernens (allen Buchwissens) zugunsten echter Weisheit, der stoischen ,ars moriendi," die im Tod ein Reifsein sieht (vgl. Shakespeares "Ripeness is all," aus Lear):

O schul! ich komme voll begier,

Die wahre weiszheit zu ergründen.

Der Tod wird so zur letzten bewuszten Handlung des Menschen, und in diesem Sinne verfolgen auch die „Märtyrer” der Gryphschen Dramen ihren Lebens- und Leidensweg. So weit die Atmosphäre friedvoller aber ernster Kontemplation; da plötzlich wechselt die Szene in das Visionäre hinüber in einem Augenblick, der sich in der Ode 
genau lokalisieren läszt, nämlich in dem Bruch zwischen der achten und der neunten Strophe:

(8) Wil hier die einsamkeit allein

Disz angenehme Werck verrichten

Und alle meine zweiffel schlichten,

Die mich umstrickt? O nein! o nein!

(9) Wie wird mir? wackelt nicht der grund, Auff dem ich steh'? rauscht ihr, o linden?

Wie? reist die erd auff ihren schlund

Und läst die wurtzeln sich entbinden?

Die stoische Lektion wird nun durch Anschauung vertieft soweit, dasz-in typisch barocker Weise-sich im Höhepunkt der Vision (Str. 22 bis 31) das Pendel zwischen grauenvoller Realistik und ruhigstoischer Ejnsicht in die Vergänglichkeit nach der Seite des makabren Elementes hin überschlägt. Das letztere Thema wird präludiert in Strophe neun und fünfzehn, dazwischen finden sich kontemplative Abschnitte (Io bis I4, I6 bis 21), die aber im Gegensatz zu der Einleitung (Str. I bis 8) eine innere Spannung aufweisen selbst da, wo Gryphius in durchaus traditionellen stoischen Ausdrucksformen spricht. Der Abschnitt Str. Io bis I4 kreist um die Thematik von der alles gleichmachenden Kraft des Todes, die wir von den Totentänzen her kennen, und Str. 16 bis 21 endet mit einer klaren Paraphrase des alten „ubi-sunt"-Themas:

Wo sind die wunder der geschöpff,

Die schönen seelen-räuberinnen?

Ich spüre nichts, als grause köpff

Und werde keiner zierath innen.

Wo sind, ob derer wissenschafft

Sich das entzückte volck entsetzet?

Die man der weissheit väter schätzet?

Die zeit hat all' hinweg gerafft.

Dann erscheinen die Verse, die gern in Anthologien abgedruckt werden:

Ich finde meistens nichts vor mir

Als gantz entfleischete gerippe.

Hirnscheitel sonder haar und zier,

Antlitzer sonder nasz' und lippe

Und häupter sonder haut und ohr,

Gesichter sonder stirn und wangen,

Die leffzen sind in nichts vergangen,

Noch wenig zähne ragen vor.

Von hier ab bleibt Gryphius im Fortissimo. „Gotisch"? Ja, unter allen Umständen. Eine Verletzung des "guten Geschmacks"? Ja, 
vom Standpunkt der Literarkritik des achtzehnten und neunzehnten Jahrhunderts, und für viele ästhetisch gerichtete Leser des zwanzigsten. Indessen, wenn auch ihrer Meinung nach Gryphius ,ein wenig stark aufträgt"-es ist doch spürbar, dasz die Angst und (fast möchte man sagen: existentielle) Erschütterung des Dichters und Darstellers solcher Szenen echt und aufrichtig ist und seine Ausdrucksweise rechtfertigt Besonders im Hinblick auf diese Verse darf Gryphius als stärkste und reinste Ausprägung des hochbarocken Seelenlebens und Todesempfindens gelten. Auf die Kirchhofsgedanken trifft ohne Zweifel Cysarz' Wort zu: „Unsere gröszten Barockdichtungen haben gleichsam Muskeln von Rubens, Gesichter von Rembrandts Hand" (a.a.O., Anm. 3, Barocke Lyrik, S. 39).

Es scheint, dasz in dieser Vision ein Seinsgrund in Gryphius erschüttert worden ist: trotz aller Widrigkeit liebte der Dichter das Leben und seine bunte Mannigfaltigkeit. Ohne diese Voraussetzung wäre ein dramatischer Aufschwung wie der in Str. 22 bis 3 I nicht möglich gewesen. Die Reaktion auf diesen Höhepunkt des Makabren erfolgt in Str. 32 und 33, in der direkten Wendung hin zu Gott. Dieses „de profundis" ist die Absage an den stoischen Glauben der Vorbereitung und Überbrückbarkeit; es ist eine Berufung in letzter Minute auf die Verheiszung der Gnade, die rational nicht erklärbar ist, und die der Mensch frei empfängt, ohne dafür etwas tun zu können oder müssen. Auf die Kirchhofsgedanken trifft Böhmes Wort vom Tod zu: "Tod aber als das Sterben bleibt das gröszte Geheimnis."

iii. Der "grosze Einsame".

Man hat Gryphius oft den ",groszen Einsamen” genannt. In der Tat begegnete er ,wie niemand um ihn in der Tiefe seines Welterlebnisses der unaufhaltsamen Zeit als einer schrecklichen, alles durchdringenden, alles vernichtenden Wirklichkeit .... In Gryphius begann die Zeit wie der Mensch zu sich selber zu kommen" (Fricke, a.a.O., Anm. 4, S. II6). Es ist die Konzentration, der gestraffte Ausdruck barocker Thematik unter Verzicht auf reine Sinnlichkeit (Gryphius schreibt wenig Liebeslyrik!), und andererseits auch auf reine Mystik, durch den sich Gryphius von seinen schlesischen Zeitgenossen unterschied. Nichts hält ihn zurück, auch aus stoischen Grenzen herauszutreten. Die Opitzsche Sprachform erhält durch ihn den tiefen seelischen Gehalt.

Wenn wir uns im übrigen Europa nach Ebenbürtigkeit umschauen, so läszt sich feststellen und nachweisen, dasz im frühbarocken Frankreich Ronsard (in seinen späteren Jahren) dem Gryphius ein wenig ähnelt. Auch hat Montaigne manchen Essay geschrieben, dessen Thematik mit der des schlesischen Dichters im Einklang steht (vgl. III, 9: De la vanité), und auch Pascal weilt im gleichen Themenbereich. Manche Stelle aus Shakespeare und Racine könnte-in Bezug 
auf die Thematik-in direkte Verbindung mit Gryphs Gedichten gebracht werden. Leider müssen wir uns mit dem Hinweis begnügen, da der Rahmen dieser Arbeit keine weiteren Ausführungen zuläszt. Unser Ergebnis kann in folgenden Worten zusammengefaszt werden:

I. In der Lyrik des Andreas Gryphius treten alle philosophischen Hauptthemen der Barockliteratur auf.

2. Diese Hauptthemen gewinnen in ihrer lyrischen Darstellung durch die formale Gebundenheit des Dichters an das Alte; im Hinblick auf das Formelement steht Gryphius zwischen Opitz einerseits, und den Dichtern des Hoch- und Spätbarock andererseits.

3. Im Vergleich zum dichterischen Schaffen des sechzehnten Jahrhunderts sieht Gryphius von der moralisierenden Tendenz eines mehr didaktisch-allegorischen Schrifttums ab; seine Lyrik (wie seine dramatischen Schöpfungen) zeigt den Menschen in einer Situation und sein Verhalten gegen von auszen auf ihn wirkende Kräfte. Die Problematik ist daher mehr metaphysisch als ethisch.

4. Gryphius glaubt an einen Sinn des menschlichen Daseins und an des Menschen Berechtigung, sich gegen den Tod zu wehren, ebenso wie an die Möglichkeit einer (direkten) Transzendenz. Er ist daher kein Pessimist.

University of Michigan.

EDELGARD E. CONRADT.

iv. Anmerkungen und bibliographische Referenzen

I. Ich zitiere nach Andreas Gryphius lyrische Gedichte, hrsg. von Hermann Palm (Tübingen, 1884), und folge der Paginierung dieser Ausgabe.

2. Der Artikel ist in: Smith College Studies in Modern Languages, XXI, 1939, p. $192 \mathrm{ff}$.

3. Herbert Cysarz, Deutsche Barockdichtung (Leipzig, 1924),

Herbert Cysarz, "Barocke Lyrik und barocke Lyriker," Einleitung zu Vor- und Frïhbarock, Deutsche Literatur in Entwicklungsreihen, (Leipzig, 1937)

Günther Müller, Deutsche Dichtung von der Renaissance bis zum Ausgang des Barock (Wildpark -Potsdam, 1927)

Paul Hankamer, Deutsche Gegenreformation und deutsches Barock (Stuttgart, 1935)

4. Gerhard Fricke, Die Bildlichkeit in der Dichtung des Andreas Gryphius (Berlin, 1933)

René Wellek, „Concept of Baroque in Literary Scholarship”, in: Journal of Aesthetics and Art Criticism, 4-5, (New York, 1945-47)

Benedetto Groce, Der Begriff des Barock (1925), auch: Storia della eta barocca in Italia (Bari, 1929).

5. Gute Ausführungen über eschatologische Prophezeiungen finden sich in:

Herbert Cysarz, Deutsche Barockdichtung (Leipzig, 1924), S. I65 (für Gryphius),

Emil Ermatinger, Weltdeutung in Grimmelshausens Simplicius Simplicissimus (Leipzig, 1925), S. $98 \mathrm{ff}$.

Emil Ermatinger, Barock und Rokoko in der deutschen Dichtung (Leipzig, 1926),

6. Dissertationes funebres, Oder Leich-Abdanckungen (Leipzig, I 666).

7. Fritz Strich, ,Die Zeit als Schicksal," in: Der Dichter und die Zeit (Bern, I947), S. 93 .

8. Walther Rehm, "Das Jahrhundert des Barock," in: Der Todesgedanke in der deutschen Dichtung vom Mittelalter bis zur Romantik (Halle, 1928), S. I92.

9. Emil Ermatinger, Barock und Rokoko (s. o. Anm. 5), S. 23. 
Io. W. Schieck, Studien zur Lebensanschauung des Andreas Gryphius (Diss. Greifswald, 1924)

Gerhard Fricke, s. Anm. 4.

II. Palm, s. O. Anm. I, S. 217 ; S. 236; S. 102; S. 135

12. Erich Trunz, ,Weltbild und Dichtung im deutschen Barock," in: Zeitschrift für Deutschkunde, LI, S. I4-30 (Leipzig und Berlin, 1937).

13. Ich folge hier den Ausführungen über die "Rangstellung des Menschen" in: Hugo Friedrich, Montaigne (Bern, 1949), S. I $49 \mathrm{ff}$.

14. Georg Rodolf Weckherlin, .Wie die Soldaten man vor zeitten/Laut mit dem mund; / So Sie ietzund / Ermahnet der Poet zu streitten".

15. Edgar Hederer, Deutsche Dichtung des Barock (München, 1954).

16. Genaueres über diese Legende und ihren Ursprung in: Karl Künstle, Die Legende der drei Lebenden und der drei Toten und der Totentanz (Freiburg i. Br., 1908).

17. Spangenberg, ein Autor aus dem Anfang des siebzehnten Jahrhunderts, läszt in seiner Tragödie Mammons Sold allen Ständen dieser Welt zur Lehr (1613) drei Jünglinge einer wunderschönen Frau entgegengehen. Sowie sie nahe vor ihr stehen, fallen dieser die Kleider vom Körper, und ein scheuszliches Gerippe mit Pfeil und Bogen steht ihnen gegenüber.

18. Als Beispiel sei gegeben Diss. Fun., S. 475: „Zuletzt stehen wir bey mehr und mehr überhand nehmenden Winter gantz entblöszet, unserer Stärcke, unsers Ansehens, unserer Wissenschafften beraubet, und beweinen, .... dasz wir in so hohem Alter nicht mehr erkennen, was wir bey sinnreichen Jahren selbst geschrieben . . . . Erreichen wir endlich den letzten Monat und Schlusz des Jahres, so werden wir wieder zu Kindern, sind untüchtig etwas weiter zu verrichten: Das Liechtt unser Augen verfällt, die Traurigkeit des Gemüths nimmt überhand, Sinn und Geist erkalten, und ist nichts mehr übrig, als ein lebendtodter Leib, der nunmehr von andern geführet, und beweget werden musz."

I9. Ronsard gibt dem Entsetzen vor dem unmittelbaren Tode einmal ganz ähnlichen Ausdruck. In seinem letzten Sonett schreibt er:

Je n'ay plus que les os, un squelette je semble,

Descharné, denervé, demusclé, depoulpé,

Que le trait de la Mort sans pardon a frappé:

Je n'ose voir mes bras que de peur je ne tremble.

(Ich zitiere nach der Ausgabe der Oeuvres Completes von Paul Laumonier, Paris, I9I 4-I942.)

\section{HEINRICH HEINES SOGENANNTE ,JOSEPHA-LIEDER".}

Bekanntlich werden das 2.6.7.8. und 9. "Traumbild" von Heines "Traumbildern" von vielen auf seine Liebe zu Josepha (Sefchen), der Tochter des Scharfrichters, bezogen, über die Heine ausführlich in seinen "Memoiren" berichtet ". So Ernst Elster in: "Heinrich Heines Buch der Lieder" 2; idem: "Heinrich Heines sämtliche Werke r. Ausgabe ${ }^{3}$. (In der 2. kritisch durchgesehenen und erläuterten Ausgabe drückt Elster sich vorsichtiger aus: „Die Traumbilder 2, 6, 7, 8, auch wohl 9, sind trotz Beyer durch Liebe zu Josepha mit beein(luszt) ${ }^{4}$. Legras möchte ebenfalls die "Traumbilder" 2, 6, 7, 8 und 9

I. Heinrich Heines sämtliche Werke. Hrsg. von Prof. Dr. Ernst Elster, Leipzig und Wien. Bibliogr. Institut VII, 502.

2. Henninger, Heilbronn, 1887 S. 9, 12.

3. S. IO, I I der Einleitung; weiter S. 13, I8, 20, 23, 28.

4. S. $43 \mathrm{I}$. 